\title{
Journalisme et publicité, entre rationalités techniques et talent personnel. Quelle place pour les « savoirs savants »?
}

Semiotic Instrumentalizations

Jean-Michel Utard

\section{OpenEdition}

\section{Journals}

Édition électronique

URL : http://journals.openedition.org/edc/202

DOI : 10.4000 /edc.202

ISSN : 2101-0366

\section{Éditeur}

Université Lille-3

\section{Édition imprimée}

Date de publication : 1 décembre 2004

ISBN : 2-9514961-5-X

ISSN : 1270-6841

Référence électronique

Jean-Michel Utard, « Journalisme et publicité, entre rationalités techniques et talent personnel. Quelle place pour les « savoirs savants »? ", Études de communication [En ligne], 27 | 2004, mis en ligne le 18 novembre 2014, consulté le 30 avril 2019. URL : http://journals.openedition.org/edc/202 ; DOI : 10.4000/edc. 202

Ce document a été généré automatiquement le 30 avril 2019.

(c) Tous droits réservés 


\title{
Journalisme et publicité, entre rationalités techniques et talent personnel. Quelle place pour les « savoirs savants »?
}

\author{
Semiotic Instrumentalizations
}

Jean-Michel Utard

1 Aussi paradoxal que cela puisse paraître, les études sur la publicité ou le journalisme ne sont pas nombreuses en sciences de l'information et de la communication. Et pourtant, l'une et l'autre de ces pratiques communicationnelles sont souvent pensées comme des prototypes de chacune des deux faces du champ : le journalisme pour l'information et la publicité pour la communication. Inversement, l'espace public est largement alimenté de représentations sur ces domaines de la vie sociale, sous forme de croyances et d'opinions, mais aussi de discours normatifs ou réflexifs. Ce décalage quantitatif entre méta-discours scientifiques et publics est d'autant plus surprenant que l'étude des médias de masse, en particulier les processus d'information, de propagande, de publicité ont constitué des terrains d'origine privilégiés pour les études de communication. Que l'on repense, par exemple, au rôle initiatique des Mythologies de Roland Barthes (1957) où la publicité et le fait divers sont promus au rang d'objets de recherche légitimes, exemplaires de la culture de masse, illustratifs des processus de production de sens, etc.

2 Nous faisons l'hypothèse que la rareté relative des travaux, sur ces objets et dans ce champ interdisciplinaire, est liée à l'abondance d'autres types de discours et aux enjeux qui les sous-tendent. Discours scientifiques, discours professionnels et discours du sens commun, ensemble, forment système. C'est dire d'une part qu'ils occupent le même espace problématique et se nourrissent les uns les autres, et d'autre part qu'ils se définissent et donc se limitent réciproquement. Le tout forme un ensemble complexe de concurrence-coopération fait de légitimation et d'instrumentalisation réciproques, d'emprunts, de controverses pas toujours académiques, mais liés par une question 
partagée : l'efficacité sociale de la communication. Qu'ils soient revendiqués ou niés, révélés ou expertisés, les effets des messages sont au centre des questionnements.

Quelle peut être alors l'autonomie d'une démarche scientifique qui se voit imposer non seulement ses catégories, mais aussi ses problématiques? Quelle peut même être son utilité alors que les pratiques professionnalisées prouvent leur savoirs par l'efficacité de leurs savoir-faire? D'un côté, pour n'être pas condamnée à n'être que la prestataire de savoirs experts ou la productrice de commentaires seconds sur des pratiques qui la précèdent, toute démarche scientifique ne peut se fonder que sur une coupure avec les savoirs construits dans d'autres logiques sociales. Mais elle ne peut trouver de légitimité sociale et de validité théorique que si elle ne rompt pas avec les enjeux qui sous-tendent les pratiques sociales qu'elle construit comme objet. Toute science des médias se trouve alors nécessairement inscrite dans les échanges que ces mêmes médias produisent et dans les interdépendances qu'ils induisent (Georgakakis, Utard, 2001).

\section{Des objets déjà construits}

4 Le rapprochement, nécessairement caricatural sous l'angle choisi, du journalisme et de la publicité n'a pas ici comme objectif de procéder à une étude comparative. Il a valeur heuristique pour interroger les interactions entre l'activité réflexive des acteurs et la construction autonome de savoirs sur la communication médiatique. À commencer par le fait que ces objets et les catégorisations auxquels ils renvoient sont imposés par plus d'un siècle d'une construction sociale qui leur donne le statut d'évidences avec lesquelles il est difficile de prendre distance.

5 Ainsi, le journalisme et la publicité semblent s'opposer aussi bien dans les définitions qui tentent d'en rendre compte que dans les réalités sociologiques de leur pratique. Ce ne sont pas les mêmes personnes qui exercent ces fonctions, leur formation est différente, les filières de leur activité sont séparées, et surtout leur inscription dans l'espace public se présente comme ayant des objectifs contradictoires. Cette opposition se retrouve dans les représentations plus ou moins modélisantes qui en sont données: la production d'informations objectives sur l'actualité événementielle du monde naturel et social pour le journaliste considéré comme le médiateur transparent du débat démocratique; la production de messages persuasifs sur les produits et les services marchands pour le publicitaire considéré comme un régulateur stratégique du marché.

6 Cette construction est indépendante du jugement de valeur que l'on peut porter sur l'une ou l'autre de ces pratiques communicationnelles. La méfiance vis-à-vis des journalistes vaut bien la publiphobie, et la croyance en l'objectivité de l'information est au moins égale au plaisir distancé que l'on prend à la réception des messages publicitaires. Même si dans leurs manifestations singulières ces pratiques et leurs productions n'ont pas la pureté du modèle, elles y renvoient en dernière analyse comme à des idéal-types censés pouvoir rendre compte de leur fonctionnement, qu'il soit conforme ou déviant.

7 C'est ce que montrent par exemple les débats que suscitent les transformations du journalisme. Qu'il s'agisse de l'apparition de nouveaux supports tels les journaux gratuits ou les magazines de marque (Ringoot, Utard, 2005), des mutations de l'information sous l'influence des logiques marchandes (Bourdieu, 1996), de la diffusion de l'information sur Internet (Damian et alii, 2001), c'est très souvent en termes de mélange des genres, de confusion des rôles, de dérive éthique que s'analysent les phénomènes observés. Plus 
généralement, c'est un mouvement de subversion du modèle informationnel par le modèle communicationnel qui sert à décrire les métamorphoses en cours.

La démarche scientifique rencontre donc des objets sociaux déjà là. Et les mouvements de réaction que suscitent les médias réactualisent périodiquement pour le chercheur les enjeux politiques, idéologiques et culturels que mobilisent ces deux modèles opposés. Ces derniers cependant ne sont pas simplement des données, mais des constructions conceptuelles affinées par une longue histoire réflexive. S'ils constituent l'opinion commune, ils sont largement produits par les acteurs, hommes ou institutions du journalisme (Ruellan, 1993) et de la publicité (Utard, 2001).

\section{Un corps de savoirs constitué}

9 Car ces deux domaines de la production médiatique ont en commun d'avoir deux faces: l'une ostensible et l'autre voilée. La première est constituée par les messages diffusés dans l'espace public, plus ou moins attribués à un auteur individuel ou collectif: une annonce, un article, un journal, etc. La seconde est le système qui permet et organise la production des messages, structure de l'ombre souvent mal connue: une agence de communication, une entreprise médiatique, une profession, etc. Ces deux versants constituent pour le journalisme comme pour la publicité deux dimensions inséparables de leur construction identitaire: d'un côté une organisation rationalisée de collecte de données et d'informations, un ensemble de techniques de leur traitement et de leur diffusion, une gestion économique de leur circulation; de l'autre côté, une boîte noire localisée dans les acteurs et qui renvoie à la mythique créativité des publicitaires ou à l'indéfinissable talent des journalistes.

10 Ces deux aspects contradictoires doivent nécessairement être affirmés ensemble. La version rationalisée contribue à fonder la croyance en l'objectivité journalistique ou l'efficacité publicitaire. La version subjective préservant la relativité des vérités en démocratie (Muhlmann, 2004) ou la dimension désirante de la matérialité du marché (Hennion et Méadel, 1997). De cette formulation, il ne faudrait pas conclure à une gestion cynique de l'image de ces métiers s'opposant à la réalité de leur exercice. L'une et l'autre dimension fondent ensemble les convictions déontologiques de ces professions, pensées à la fois comme activité collective et comme acte individuel.

11 C'est le côté rationnel qui fait l'objet de formalisations élaborées essentiellement à des fins de formation et que l'on trouve le plus systématiquement exposé dans des manuels, dont les archétypes sont Le publicitor de Brochand et Lendrevie (1983), et le Manuel de journalisme d'Agnès et Hervouet (2004). Car la professionnalisation suppose la constitution d'un corps de connaissances transmissibles aux aspirants à la fonction. Cette part formalisée s'est même construite comme un acte de rupture avec les pratiques empiriques ou d'amateurs (Chessel, 1998; Palmer, 1983). S'il reste cependant une part de la compétence individuelle qui relève de l'ineffable et qui "ne s'apprend pas » (Georgakakis, 1995), elle consiste tout autant dans la capacité à catalyser les règles collectives qu'à les transgresser.

12 En même temps que s'élabore un corps de savoirs, se constituent des identités professionnelles qui trouvent leur traduction dans la formation de corps de métiers (Ruellan, 1997). Et du même geste, ces pratiques sociales se naturalisent comme fonctions nécessaires de la société démocratique et marchande. Cette autodéfinition par les acteurs 
n'est pourtant pas totalement endogène. Ceux-ci trouvent leur consécration en s'imposant comme composante nécessaire, sinon exclusive de la médiation sociale. Cette reconnaissance se matérialise en un ensemble d'actes législatifs et juridiques, et de références déontologiques, dont le degré d'institutionnalisation différencie ces deux domaines professionnels. Sans constituer un ordre à l'instar des médecins ou des avocats, le journalisme s'est construit en définissant ses normes d'entrée et d'appartenance mises en œuvre par un organisme spécifique (la commission de la carte de presse), alors que la publicité relève du droit commercial pour ce qui est de l'exercice de la profession et la régulation des relations contractuelles interprofessionnelles. Tout en revendiquant l'application du principe de la liberté d'information, l'un et l'autre se réfèrent à des cadres déontologiques dont le Bureau de vérification de la publicité (BVP) surveille l'application pour les publicitaires, et que spécifient les chartes rédactionnelles des entreprises de presse pour les journalistes (Prodhomme, 2003). Les professions journalistique et publicitaire se fondent donc sur une sorte de contrat social dont les règles plus ou moins explicitées contribuent à en restreindre l'accès et à en contraindre l'exercice.

13 Par ailleurs, ces pratiques cherchent une part de leur crédibilité et de leur légitimité dans les rapports qu'elles entretiennent avec des savoirs de type scientifique (Neveu et Rieffel, 1991). Ici, journalisme et publicité se distinguent.

\section{Le rapport instrumental de la publicité à la science}

14 La publicité tient toujours un double discours vis-à-vis des savoirs de type scientifique. «Les publicitaires ne sont ni des 'saltimbanques', ni des 'géomètres'. Mais tout de même un petit peu de chaque » (Brochand, 1983, p. 8). Elle se présente tour à tour, en fonction des interlocuteurs et de la conjoncture, comme une pratique scientifiquement fondée ou comme un ensemble de savoir-faire construits par l'expérience. La créativité elle-même, cette part mythique de l'activité que nous évoquions ci-dessus, n'échappe pas à ce balancier entre un ensemble d'opérations formalisables, voire scientifiquement analysables, et une opération magique de transmutation d'une valeur d'usage en objet de désir.

15 Si le discours public est relativement silencieux sur la machine publicitaire, les métadiscours sur les méthodes et les théories sont abondants dans les manuels de formation et les ouvrages "pratiques » à destination des acteurs des entreprises. Les références aux disciplines académiques y sont explicites (économie, gestion, psychologie, sociologie, sémiologie), avec exposés de théories et bibliographies, même si le spectre des unes et des autres est assez limité et redondant. Plus généralement, et ceci depuis plus de cinquante ans, il est admis que la publicité constitue « une expérimentation et une clinique dans un vaste champ économico-psycho-social », et donc qu'elle relèverait d'une démarche scientifique (Morin, in Cathelat, 1968).

De fait, c'est moins le type et le nombre de références qui nous retiennent ici que la forme d'importation des savoirs savants. Ceux-ci sont coupés des conditions de leur élaboration, et les conclusions provisoires de l'argumentation scientifique sont considérées comme des représentations vraies $\mathrm{du}$ monde. Les rationalisations professionnelles sont remodelées en fonction des évolutions et des transformations du savoir scientifique. Par exemple, la publicité se fait tour à tour "béhavioriste", "motivationniste ", " psychanalytique », etc. au gré des théorisations scientifiques (Bonnange, Thomas, 1987 ; 
Haineault, Roy, 1984). Ces variantes, qui coexistent à une même époque, alimentent la concurrence entre acteurs du champ, fournissant aux «philosophies» des agences les arguments pour faire valoir leur différence.

Peu importe cependant cette réduction positiviste des sciences. Celles-ci n'ont qu'une faible vertu opératoire dans l'activité quotidienne des publicitaires. Et les budgets alloués permettent rarement la mise en œuvre des procédures lourdes et coûteuses d'enquêtes, d'études et de tests. Comme production de messages destinés à induire des préférences auprès de consommateurs ciblés, la pratique professionnelle est le plus souvent affaire d'expérience, d'intuition et de routine. Elle repose sur d'une représentation syncrétique de l'action de la publicité: une intervention pour modifier les processus cognitifs, affectifs et comportementaux de consommateurs sociologiquement situés, en utilisant les moyens des communications de masse. Point n'est besoin alors aux acteurs de maîtriser des savoirs, ni de mettre en œuvre des démarches scientifiques. Il leur suffit de savoir que leur pratique empirique est scientifiquement assurée, voire prouvée.

\section{La mise à distance de la science par le journalisme}

18 L'apparition de la presse de masse au $\mathrm{XIX}^{\mathrm{e}}$ siècle a transformé la nature même du journalisme. S'éloignant de sa fonction d'expression des opinions, le journal est devenu le lieu de présentation des faits. Suivant l'élargissement de leur public, les journaux ont étendu le territoire de leur action et le spectre de leurs préoccupations, transformant les journalistes en collecteurs d'informations toujours plus abondantes et diversifiées. L'activité journalistique s'est ainsi industrialisée, faisant de l'information une production marchande, fruit d'une division du travail et d'une rationalité technique.

19 En France, cette transformation est présentée comme une rupture, correspondant de fait à une « invention du journalisme » (Ferenczi, 1993). Quittant la position d'acteur dans le monde politique ou d'auteur dans le monde littéraire, le journalisme s'est professionnalisé en élaborant un ensemble de techniques de recueil et de traitement de l'information. Celles-ci, articulées autour de l'opposition du fait et du commentaire, visent à produire un discours objectif, compris à la fois comme vrai et honnête.

Car en plus d'un métier, le journalisme est pensé comme une fonction de la démocratie. À l'inverse de l'action des publicitaires, son intervention exclut toute visée stratégique d'influence. Bien plus, le journalisme revendique son autonomie par rapport à ses sources et ses publics, aussi bien que par rapport aux stratégies des entreprises de presse. La déontologie est présentée comme "un réglage anticipé des relations à l'égard de ces destinataires de la pratique journalistique que sont les lecteurs, les pairs et les 'sources' » (Siméan, 1992, p. 38). Une telle posture implique la mise à distance de toute maitrise scientifique de ces relations, qui assimilerait le journalisme à une activité de propagande (Georgakakis, Utard, 2001). Et les formalisations de cette pratique relèvent toujours d'une rationalité limitée, associant généralisation de l'expérience et talent individuel (Ringoot, 2005).

21 Les rationalisations professionnelles placent ainsi l'individu journaliste au centre de la pratique et implique un rapport à la réalité alternatif à la démarche scientifique. La science, dans la mesure où elle vise à construire des modèles explicatifs pour rendre compte des relations entre les phénomènes, est accusée de produire des artefacts qui réduisent et transforment la perception $\mathrm{du}$ réel. Sans refuser toute valeur de vérité aux 
constructions scientifiques, le journalisme se pose en démarche concurrente de production de savoirs vrais sur le monde. En se plaçant résolument dans le présent de l'actualité, le journalisme prétend s'en tenir à la singularité des phénomènes et ne produire que des "faits", évitant toute généralisation abusive. Valorisant le contact direct avec le terrain, il oppose sa démarche d'enquête, tournée vers la quête d'authenticité, à celle de la sociologie, visant la vérité statistique.

Les nombreux travaux de sociologie du journalisme (Neveu, 2001) montrent qu'une telle vision du métier relève plus d'une mythologie professionnelle (Le Bohec, 2000) que de la description des pratiques concrètes. Par exemple, le journalisme lui-même a abondamment recours aux techniques du sondage d'opinion dont les méthodes contribuent à informer le réel, loin de la seule perception directe du journaliste. D'autre part, son rapport aux faits est largement dépendant des constructions sociales de la réalité produites en dehors de la sphère journalistique elle-même. Le journaliste croise ainsi les versions du réel produites par les acteurs sociaux. Ce sont aussi toutes sortes de représentations du monde construites par la science qui contribuent à orienter la perception de la réalité naturelle et sociale, et qui imprègnent le discours journalistique. Et les informations stratégiquement élaborées par des acteurs visant à influencer l'opinion dans le sens de leurs intérêts, font de ces derniers des sources "professionnalisées » (Schlesinger, 1992). Enfin, les journalistes eux-mêmes, dont une très grande majorité travaille dans la presse magazine, ont fait de leur relation aux lecteurs et de la réponse à leurs attentes, une modalité de leur savoir-faire. Les techniques du marketing de presse rapprochent ici les pratiques journalistiques de celles des publicitaires.

\section{Une science des médias « intimidée »}

En incluant la science dans son dispositif, à la fois comme stock de connaissances et comme procédure expérimentale, la publicité se présente comme une activité de production symbolique impersonnelle. Les acteurs n'y sont identifiés que sous les noms de quelques héros dont les écrits et les manifestations publiques ont eu essentiellement pour fonction de légitimer une activité méconnue (Bleustein-Blanchet, 1970) ou mal perçue (Séguéla, 1979). Mais les bataillons de l'ombre attendent encore les sociographes d'une profession qui feront apparaître la diversité des situations, les hiérarchies, les routines, les approximations d'une production rhétorique qui vise avant tout à mettre une information commerciale à disposition d'un public de consommateurs. Quarante ans après l'analyse de la publicité Panzani par Roland Barthes (1964), l'étude scientifique de la publicité reste centrée sur le message, que ce soit pour en évaluer la pertinence sémiologique, en démonter le fonctionnement idéologique ou en mesurer l'efficacité perceptive ou mémorielle. Même remarquée, une annonce publicitaire n'a pas d'auteur et renvoie presque exlusivement à la responsabilité énonciative de l'annonceur. Le processus de production, quand il est pris en compte, reprend sans trop de distance critique l'exposé des procédures telles qu'elles sont formalisées dans les manuels (la fameuse copy-stratégie et ses variantes), même si celles-ci se démarquent à peine, sous le jargon anglo-saxon, des exposés normatifs de la rhétorique ancienne. En somme, la mise en scène scientifique de la volonté persuasive universalise un processus de production sous la forme d'une énonciation impersonnelle dont seule l'approche critique pourrait constituer une démarche scientifique autonome. 
24 A l'inverse, et malgré la mise en évidence de l'industrialisation du processus de production de l'information, les recherches sur le journalisme se caractérisent par un intérêt accru pour les acteurs en situation et le rôle des interactions dans la pratique professionnelle des journalistes. La synthèse par Erik Neveu (2001) des recherches de sociologie empirique dans ce domaine montre qu'il est difficile de continuer à parler du journalisme au singulier. Ainsi le modèle de la médiation de l'information dans un espace public rationnel et unifié est largement contrebalancé par la diversité des objets, des statuts, des spécialisations, des hiérarchies, des modalités de travail, etc. qui font éclater l'identité professionnelle du journalisme. L'information elle-même ne peut plus être pensée comme un objet unique, mais bien comme le produit segmenté de pratiques sociales diversifiées. Cependant, cet intérêt pour les logiques d'acteurs, y compris dans l'étude des interactions fines d'une microsociologie compréhensive des pratiques, oriente les interrogations vers des questions éthiques au détriment de celle des processus sociaux structurants. La problématique du discours elle-même se voit confinée à un processus énonciatif où la pratique discursive se résume au choix des ressources linguistiques et rhétoriques propres à manifester le mieux possible ces normes éthiques, telle l'objectivité journalistique.

Les transformations de la production et de la diffusion d'information, accélérées par le développement des nouvelles technologies de l'information et de la communication, posent à nouveaux frais la question des catégories d'analyse produites dans l'espace professionnel. En particulier, les phénomènes de convergence observés (Damian et alii, 2001) dans les stratégies des acteurs, dans l'identité des professionnels, dans les produits informationnels et communicationnels ne peuvent plus être pensés en termes de confusion ou de brouillage par rapport à des modèles largement idéalisés. La posture critique elle-même, qui est une dimension d'une approche scientifique, ne peut être sauvergardée qu'en échappant aux catégorisations produites par les acteurs, dont les logiques sont largement déterminées par les objectifs de fermeture et de reproduction de leur champ professionnel.

\section{BIBLIOGRAPHIE}

Agnès, Yves \& Hervouet, Loïc, (2004), Manuel de journalisme, La Découverte.

Barthes, Roland, (1957), Mythologies, Seuil.

Barthes, Roland, (1964), « Rhétorique de l'image », Communications $n^{\circ} 4$.

Bleustein-Blanchet, Marcel, (1970), La rage de convaincre, Robert Laffont.

Bonnange, Claude \& Thomas, Chantal, (1987), Don Juan ou Pavlov. Essai sur la communication publicitaire, Le Seuil, coll. Points/Essais.

Bourdieu, Pierre, (1996), Sur la télévision, (suivi de) L'emprise du journalisme, Liber.

Brochand, Bernard \& Lendrevie, Jacques, (1983), Le publicitor, Dalloz, (et rééditions). 
Cathelat, Bernard \& Cadet, André, (1976), Publicité et société. De l'instrument économique à l'institution sociale, Petite Bibliothèque Payot, (Préface d'Edgar Morin à la première édition, $\mathrm{La}$ Publicité, Payot, 1968).

Chessel, Marie-Emmanuelle, (1998), La publicité. Naissance d'une profession, 1900-1940, CNRS Éditions.

Damian, Béatrice et alii, (2001), Information, locale. Le paysage médiatique à l'ère électronique, L'Harmattan, coll. Communication et civilisation.

Ferenczi, Thomas, (1993), L'invention du journalisme en France, Payot.

Georgakakis, Didier, (1995), « Comment enseigner ce qui ne s'apprend pas. Rationalisations de la 'communication de masse' et pratiques pédagogiques en école privée », Politix 29.

Georgakakis, Didier \& Utard, Jean-Michel, (dir.), (2001), Science des médias. Jalons pour une histoire politique, l'Harmattan.

Haineault, Doris-Louise \& Roy, Jean-Yves, (1984), L'inconscient qu'on affiche, Aubier.

Hennion, Antoine \& Méadel, Cécile, (1997), «Les ouvriers du désir. Du produit au consommateur, la médiation publicitaire ", Sociologie de la communication, pp. 593-619.

Johanna, Siméan, (1992), « Déontologie et crédibilité : le réglage des relations professionnelles au Centre de formation des journalistes », Politix n¹9, pp. 37-55.

Le Bohec, Jacques, (2000), Les mythes professionnels des journalistes, L'Harmattan.

Muhlmann, Géraldine, (2004), Une histoire politique du journalisme, XIX ${ }^{e}-X X^{e}$ siècle, P.U.F.

Neveu, Erik \& Rieffel, Rémy, (1991), « Les effets de réalité des sciences de la communication », Réseaux 50, pp. 13-37.

Neveu, Erik, (2001), Sociologie du journalisme, La Découverte/Repères, (nouvelle édition, 2004).

Palmer, Michaël, (1983), Des petits journaux aux grandes agences. Naissance du journalisme moderne, Aubier.

Prodhomme, Magali, (2003), La place du discours sur l'éthique dans la construction de l'identité et de l'espace professionnels des journalistes, Thèse de doctorat (dir., Jean-François Tétu), Lyon 2.

Ringoot, Roselyne \& Utard, Jean-Michel, (à paraître en 2005), L'invention permanente du journalisme (titre provisoire), Presses Universitaires de Rennes, coll. Res Publica.

Ringoot, Roselyne, (dir.), (à paraître en 2005), Genres journalistiques. Savoirs et savoir-faire, actes du colloque de Lannion, septembre 2004, L'Harmattan.

Ruellan, Denis, (1993), Le professionnalisme du flou, Presses universitaires de Grenoble.

Ruellan, Denis, (1997), Les « pros » du journalisme. De l'état au statut, la construction d'un espace professionnel, Presses universitaires de Rennes.

Schlesinger, Philip, (1992), « Repenser la sociologie du journalisme. Les stratégies de la source d'information et les limites du média-centrisme », Réseaux n51, pp. 75-98.

Séguéla, Jacques, (1979), Ne dites pas à ma mère que je suis dans la publicité... Elle me croit pianiste dans un bordel, Flammarion.

Utard, Jean-Michel, (1999), « Les métiers de la communication : des techniques sans technologie? », in : Science et Technologie : regards croisés, Actes du colloque CNRIUT, L'Harmattan. 
Utard, Jean-Michel, (2001), « Les publicitaires : rationalités techniques et imaginaires professionnels », Télévision et publicité, INA/Dossiers de l'audiovisuel n99, octobre.

\section{RÉSUMÉS}

Les sciences de la communication sont hantées par deux écueils : celui de l'expertise, qui les enferme dans les logiques d'acteurs, et celui de la position critique, qui les isole de la réalité des processus sociaux. Le journalisme et la publicité constituent deux domaines où le poids des rationalités professionnelles pèsent sur leur approche scientifique. Emprise sur la réalité des pratiques et distance par rapport aux stratégies d'acteurs suppose une interrogation critique de ces rationalisations.

\section{INDEX}

Mots-clés : journalisme, publicité, identité professionnelle, science des médias

Keywords : Journalism and Advertising, between Technical Rationalities and Personal Talent. What Place for "Scholarly Knowledge"?

\section{AUTEUR}

\section{JEAN-MICHEL UTARD}

Jean-Michel Utard est maître de conférences en Sciences de l'information et de la communication. Animateur du Groupe de recherche en analyse du discours des médias (GRAM) au sein de la SFSIC.

L'enseignement de la publicité et de la communication d'entreprise fonde ses réflexions sur les rationalisations des pratiques professionnelles et leur lien avec les savoirs savants. Ses recherches portent sur le discours journalistique et ses transformations, dans une perspective mettant en rapport la pratique discursive des journalistes avec les territoires et les mutations professionnelles, organisationnelles et technologiques de son exercice. 